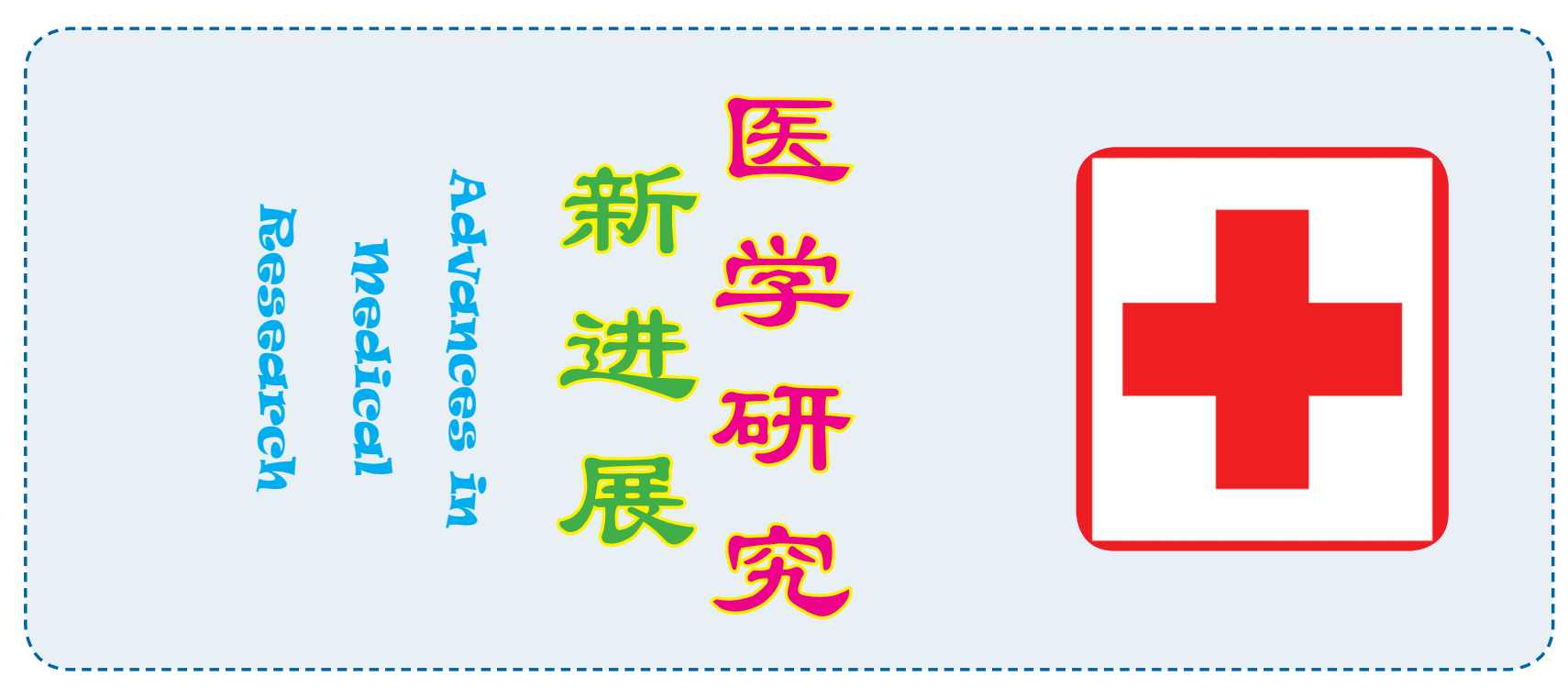

\title{
喝甘菊茶降低 患甲状腺癌的风险
}

\section{张世炳 \\ 武汉华易研生物科技有限公司}

最近, 一份病例对照研究首次指出, 长期喝草 药茶 (特别是甘菊茶) 可能降低患甲状腺癌及其他甲 状腺疾病的风险。该研究由雅典预防医学研究所的 研究团队完成, 结果发表在最新一期的 European Journal of Public Health杂志上。

茶是世界上被最广泛饮用的, 仅次于水的饮料 之一。之前已有研究证实喝茶可能起到降低癌症风 险的作用, 但却极少有研究深入到内分泌肿瘤的层 面。这项最新的研究纳入了 113 例已通过组织病理 学证明患有甲状腺癌的患者, 以及与之相匹配的 138 名健康患者对照; 同时, 该研究还纳入了 286 例患有 良性甲状腺疾病的患者, 以揭示喝茶与一般甲状腺 疾病之间的联系。

根据饮茶的频率和持续时间: 无饮茶习惯、每 周至多 1 次、每周 2 至 6 次、或每天至少 1 次; 以及 不足 1 年、 15 年以内、 15 至 30 年、或 30 年以上, 研究团队将参与者进行了分类、统计。

结果显示, 随着饮茶 (甘菊茶) 频率的增加, 患恶性、良性或其他任何类型甲状腺疾病的风险都 显著降低, 该结果在校正年龄、性别和身高体重指数
(BMI) 参数后，仍具有非常显著的关联性。

相对于那些不饮茶的人, 每周喝茶 2 至 6 次的 参与者患甲状腺癌的风险要低得多, 该结果不受吸 烟、饮酒和咖啡等因素的明显影响。研究还发现, 随着饮甘菊茶年限的增加, 患恶性和良性甲状腺疾 病的几率均显著降低, 尤其是那些饮茶超过 30 年的 参与者。除了甘菊茶, 高山茶和鼠尾草茶对患者也具 有保护效应，但没有甘菊茶的效果明显。

作者推测, 喝茶降低患良性和恶性疾病的潜在 机制可归因于茶含有多酚类, 类黄酮和儿茶酚活性 成分, 源于它们所具有的抗氧化、抗炎和抗肿瘤活性。 作者最后也指出, 尽管联系显著, 仅通过病例对照 研究尚不能建立明确的因果关系。因此, 还需更多 的临床和基础研究来进一步证实该流行病学调查所 发现的结果, 以及揭示饮茶所蕴含的疾病保护机制。

编注: 甘菊茶 (Chamomile Tea), 又叫洋甘菊茶, 多以西式茶的形式出现。甘菊茶饮品迄今已有上千 年的历史, 具有抗衰老和凝神静气的作用, 长期饮 用有助于排除体内毒素、消炎、美白润肤, 还能适 当缓解压力, 帮助睡眠, 是西方注重健康人群的首 选饮品。现在, 我们又发现了一项新的功效——长 期饮用甘菊茶可降低患甲状腺疾病的风险!

\section{参考文献}

[1] Riza E, Linos A, Petralias A, de Martinis L, Duntas L, Linos D, The effect of Greek herbal tea consumption on thyroid cancer: a case-control study. Eur J Public Health, 2015;25(6):1001-1005. doi: 10.1093/eurpub/ckv063. 